\title{
NIDDK initiatives addressing health disparities in chronic diseases
}

\section{B. Tibor Roberts and Griffin P. Rodgers}

The National Institute of Diabetes and Digestive and Kidney Diseases, NIH, Bethesda, Maryland, USA.

A of the COVID-19 pandemic, we have witnessed heartbreaking events that highlight racial injustice in our country. The weight of racial injustice falls heavily on people burdened by health disparities and likely contributes to the increased morbidity and mortality of COVID-19 in minority populations. Additionally, COVID-19-related disparities and effects are exacerbated by chronic diseases, such as obesity, diabetes, and kidney disease, that disproportionately affect US minority groups. Because combating these conditions is central to the mission of the National Institute of Diabetes and Digestive and Kidney Diseases (NIDDK), we are firmly committed to research programs aimed at reducing COVID-19 disparities, such as Rapid Acceleration of Diagnostics-Underserved Populations (1). Moreover, because many NIDDK mission diseases place disparate burdens on minority groups and people with limited resources, and because we believe health equity is integral to social justice, the NIDDK has reaffirmed its commitment to combating health disparities - whether pandemic related or not - through basic, translational, and clinical research $(2,3)$.

\section{NIDDK approaches to alleviating health disparities in its mission diseases}

Here we describe four major avenues that the NIDDK is pursuing toward that goal. First, the Institute pledges to continue vigorous efforts to recruit diverse study cohorts inclusive of those most affected, which often means reaching out to underserved segments of the population. Second, because having people from disproportionately affected backgrounds conduct and guide research helps address health disparities most effectively, the NIDDK seeks to open doors for young people from underrepresented groups through training, support, and inspiration to pursue research careers. Indeed, a pipeline of new ideas and of invaluable, often untapped scientific talent will benefit our society in countless ways. Third, engaging clinical trial participants more broadly in the research enterprise can help advance scientific inquiry; thus, the NIDDK is now implementing strategies to promote participant engagement, not only as study volunteers, but also in study design, recruitment, and consent. Fourth, the Institute is supporting research to identify the causes of health disparities, fueling clinical research by yielding testable hypotheses. Disparities may stem partly from biological differences, such as risk alleles more prevalent in one population than in another. Disparities that stem from systematic differences in access to care, environmental exposures, and other external factors are of equal interest; for this reason, the NIDDK also supports research on social determinants of health, which is crucial for lighting a path toward health equity and a vital complement to studying the biological etiology of disparities.

Mutually reinforcing approaches to addressing health disparities

The four approaches outlined above can interact and support one another, as illustrated by the NIDDK-supported APOL1 Long-term Kidney Transplantation Outcomes Network (APOLLO). Groundbreaking NIDDK research demonstrated a key biological factor contributing to the elevated rates of end-stage renal disease (ESRD) in people of African descent: specific alleles of the APOL1 gene found almost exclusively in people of recent African ancestry are associated with significantly elevated
ESRD risk. Based on this discovery, the NIDDK led the development of the APOLLO research network to determine the impact of $A P O L 1$ genetic variants on kidney transplant outcomes for both living African American donors and recipients (4). The NIH's National Institute on Minority Health and Health Disparities and National Institute of Allergy and Infectious Diseases are valued collaborators in this effort. The cohort of 2614 donor-recipient pairs, being recruited through 13 APOLLO study sites, will potentially allow assessment of outcomes from nearly all transplanted kidneys from African Americans in the nation during the study.

APOLLO is also pioneering the Institute's approach to participant engagement via its Community Advisory Council (CAC). Composed of African American transplant recipients, kidney donors, and individuals on dialysis, the CAC provides input and guidance on study design, including recruitment, retention, implementation of protocols, and return of results. Members participate in regular conference calls, and some participate in Steering Committee meetings. Thus, APOLLO integrates three approaches to overcoming disparities: it proceeds from a seminal discovery about a health disparity's cause, is composed of a large and representative group of those most affected, and is demonstrating the value of engaging participants in research.

\section{The NIDDK's commitment to recruiting representative study cohorts}

Studying etiology and treatment in those most affected has long been a major consideration for the NIDDK in designing interventional and epidemiological studies. For example, because the heavy burden of renal disease African Americans bear is apparent long before progression to ESRD, they comprise $45 \%$ of participants in the Chronic Renal Insufficiency Cohort Study, which is examining risk factors for 
progression of chronic kidney disease (CKD) and cardiovascular disease in people with CKD (5).

The Glycemia Reduction Approaches in Diabetes: A Comparative Effectiveness Study is a multicenter randomized controlled trial sponsored by the NIDDK evaluating four diabetes medications added to metformin in people with type 2 diabetes, among whom $20 \%$ of participants are non-Hispanic Black and 18\% are Hispanic/Latino (6). NIDDK's intramural Phoenix Epidemiology and Clinical Research Branch has identified important environmental and genetic determinants of type 2 diabetes in the Pima Indians of Arizona (7), who have among the highest rates of the disease in the world, and facilitated American Indian participation in clinical trials. The NIDDK's long-standing commitment to overcoming barriers to inclusion for participants from disproportionately affected groups is clear from landmark NIDDK-led clinical research, such as the Diabetes Prevention Program (DPP) and the DPP Outcomes Study. Because minority participants comprised more than $45 \%$ of the cohort, the researchers were able to demonstrate not only that the DPP lifestyle intervention and treatment with metformin were both capable of preventing type 2 diabetes in adults at risk for the disease but also that the benefits of the interventions were generalizable, helping African American, Hispanic, American Indian, Asian, and White participants equally (8).

Indeed, because the disparate burdens of pediatric type 2 diabetes are particularly acute, nearly $80 \%$ of study participants in the Treatment Options for Diabetes in Adolescents and Youth (TODAY) trial were from highly affected minority groups. Subgroup analyses were therefore able to demonstrate that in people with type 2 diabetes who are 20 years old or younger, metformin alone was particularly ineffective for maintaining blood glucose control among participants identifying as non-Hispanic Black (9). The TODAY results underscore the enormous importance of finding effective approaches for treating and preventing pediatric type 2 diabetes: because the disparities in its prevalence are dramatic, such improvements in care would almost certainly translate to improved health equity.

\section{Promoting diversity in the scientific workforce}

While NIDDK researchers from every race and ethnicity are striving to reduce health disparities, we believe that these vital efforts would benefit substantially from having a scientific workforce that better reflects the diverse backgrounds and experiences of the US population. Indeed, the overall biomedical research enterprise would be greatly strengthened by the scientific ideas and talent of people currently underrepresented in research. While scientific talent is surely well represented across all groups, opportunity is not. We must therefore ensure that young people are exposed to the challenges, joys, and opportunities of science and are provided needed mentoring.

The NIDDK's approach to overcoming the dearth of minority scientists therefore begins before most young people have chosen a profession and continues through support and mentorship for minority investigators early in their careers. For example, each year the High School Short-Term Research Experience for Underrepresented Persons (STEP-UP) program provides a stimulating, rigorous 8- to 10-week summer opportunity to discover experimental science for about 100 African American, Latino/Hispanic, and American Indian or Alaska Native students, as well as students from US territories (10). STEP-UP also supports undergraduates interested in conducting NIDDK-supported research. In both segments of the program, students are encouraged to work with mentors near their home or school. Complementing this effort, the NIDDK Diversity Summer Research Training Program (DSRTP) brings college students from minority groups to the NIH campus for mentored, 10-week research experiences. Both DSRTP and STEP-UP are intended to help build and sustain a diverse biomedical, behavioral, clinical, and social science researcher pipeline focused on NIDDK mission areas. NIDDK support for minority investigators continues through graduate school and into faculty positions. For example, the Network of Minority Health Research Investigators (NMRI) connects postdoctoral and junior faculty investigators with more senior researchers who mentor and serve as role models for them. NMRI, though supported by the NIDDK, is "owned" by its members, who maintain the program in part through recruitment of new members. Details of these and other NIDDK programs designed to increase the number of minority investigators - and foster their success - may be found on the NIDDK website (11).

\section{Conclusions}

Systemic changes across all sectors of our country will be needed to achieve health equity. While many changes are beyond the missions of the NIDDK and NIH, we believe that research will play a key role. The programs described here are just a sample of the NIDDK's ongoing work toward that goal, and we will continue striving to ensure the research we support benefits all of America - especially those most burdened by the diseases in our mission.

\section{Acknowledgments}

We thank Heather Rieff, Lisa Gansheroff, Robert A. Star, and Lawrence Agodoa for their helpful input and thoughtful discussion.

Address correspondence to: Griffin P. Rodgers, NIDDK, NIH, Building 31, Room 9A52, Bethesda, Maryland 20892-2560, USA. Phone:301.496.5741; Email: gr5n@nih.gov.

1. NIH. Emergency Awards: RADx-UP Coordination and Data Collection Center (CDCC) (U24 Clinical Trial Optional). https:// grants.nih.gov/grants/guide/rfa-files/RFAOD-20-013.html. Updated June 26, 2020. Accessed July 21, 2020.

2. NIH. Notice of Special Interest (NOSI): addressing health disparities in NIDDK diseases. https:/grants.nih.gov/grants/guide/notice-files/ NOT-DK-20-003.html. Updated December 16, 2019. Accessed July 21, 2020.

3. NIH. Patient-oriented research to mitigate health disparities and lessen the burden of chronic diseases within the mission of NIDDK. https://grants.nih.gov/grants/guide/rfa-files/ RFA-DK-20-015.html. Updated March 10, 2020. Accessed July 21, 2020.

4. Freedman BI, et al. APOL1 Long-term Kidney Transplantation Outcomes Network (APOLLO): design and rationale. Kidney Int Rep. 2020;5(3):278-288.

5. Lash JP, et al. Chronic Renal Insufficiency Cohort (CRIC) Study: baseline characteristics and associations with kidney function. Clin J Am Soc Nephrol. 2009;4(8):1302-1311.

6. Wexler DJ, et al. Baseline characteristics of randomized participants in the Glycemia Reduction Approaches in Diabetes: A Comparative Effectiveness Study (GRADE). Diabetes Care. 2019;42(11):2098-2107. 
7. Staimez LR, Deepa M, Ali MK, Mohan V, Hanson RL, Narayan KMV. Tale of two Indians: heterogeneity in type 2 diabetes pathophysiology. Diabetes Metab Res Rev. 2019;35(8):e3192.

8. Knowler WC, et al. Reduction in the inci- dence of type 2 diabetes with lifestyle intervention or metformin. $N$ Engl J Med. 2002;346(6):393-403.

9. TODAY Study Group, et al. A clinical trial to maintain glycemic control in youth with type 2 diabetes. N Engl J Med. 2012;366(24):2247-2256.
10. Rivers R, et al. The NIDDK High School ShortTerm Research Experience for Underrepresented Persons. Ethn Dis. 2020;30(1):5-14.

11. NIDDK. Diversity programs. https://www.niddk. nih.gov/research-funding/research-programs/ diversity-programs. Accessed July 21, 2020. 